\title{
Distribution and Sharing of e-Learning Courseware by Weighted Keywords
}

\author{
Takao Shimomura, Kenji Ikeda, and Adriano Montanaro
}

\begin{abstract}
Web-based training is popular around the world, where a large number of people can study at the same time through the Internet anytime and anywhere. The major issue of current e-learning is courseware distribution between different platforms. SCORM (sharable content object reference model) is one of the solutions for courseware sharing. However, to make SCORM-conformable courseware, some knowledge about HTML and JavaScript is required. We have developed a SWF (Sharable Web Fragment)-based e-learning system, where courseware is created with sharable Web fragments such as Web pages, images and other resources, and the courseware can be distributed to another platform by export and import facilities. The exported meta-information is architecture-independent and provides a model of courseware distribution. This paper presents how to promote the distribution and sharing of Web-based e-learning courseware, where courseware can be easily retrieved by weighted keywords.
\end{abstract}

Index Terms-Courseware, distribution, learning, sharing, web.

\section{INTRODUCTION}

Web-based training is popular around the world, where a large number of people can study at the same time through the Internet anytime and anywhere. There are no limitations of time and space with e-learning that uses a Web browser. The contents of courseware are presented to learners using multimedia such as text, diagrams, images, animation, music, and videos. They can check the results of their study by themselves because the score is displayed after questions are answered. For example, Moodle [1] is used as an online infection prevention and control program [2] for medical students. A Web-based e-learning platform [3] for physical education provides sports related courseware which includes physical motions, exercise rules and first-aid treatment. The courseware is represented using digital multimedia materials which include video, 2D animation and 3D virtual reality. Autonomous learning [4] plays an important role in raising students' learning effect and innovation ability. W. Song, et al. developed a system that consists of a management system, a learning system, a homework system and an interactive answering system. Semantic Web technology [5] in e-learning offers various semantic-based services to the students and the instructors. Students can perform semantic

Manuscript received March 3, 2014; revised July 7, 2014.

Takao Shimomura and Kenji Ikeda are with the University of Tokushima, Tokushima 770-8506 Japan (e-mail: simomura@is.tokushima-u.ac.jp, ikeda@is.tokushima-u.ac.jp).

Adriano Montanaro is with the University of Padova, Padova, Italy (e-mail: montanaro@dmsa.unipd.it). querying for learning materials.

The major issue of current e-learning is the difficulty of resources sharing and searching, which is mostly caused by the weak-semantic learning resources and the poor sharing mechanism. To solve this problem, for example, by applying ontology and Web services technology [6] to e-learning, the learning resources are well defined and information exchanging is supported. AICC [7] develops guidelines for aviation industry in the development, delivery, and evaluation of computer-based training and related training technologies. It assists airplane operators in development of guidelines which promote the economic and effective implementation of computer-based training media and develops guidelines to enable interoperability. Qu and Nejdl [8] re-designed all learning resources contained in an existing Java course according to SCORM (Sharable Content Object Reference Model) [9] so that they can be exchanged between different learning management systems. A Web-based SCORM run-time environment was also implemented using JSP, Java Servlet, and JavaScript, which is able to dynamically render course structure into its corresponding Web presentation on the basis of predefined SCORM content packaging application profiles. However, to make SCORM-conformable courseware, some knowledge about HTML and JavaScript is required. Li and Zhang [10] failed to add some useful resources or contents from SCORM to Moodle [1] because of the lack of interoperability between SCORM and Moodle. They needed to implement adding, editing, deleting and moving those resources in Moodle.

On the other hand, WebOL [11] creates some questions based on Web pages and packages them so that they can be available in another platform. It generates HTML and JavaScript code and maintains all resources in a single directory ready for distribution. A project can be run from a Web browser by opening the start-up page created by WebOL. However, it does not contain any features for the recording of assessment results.

The authors have developed a SWF (Sharable Web Fragment)-based e-learning system [12], [13], where courseware is created with sharable Web fragments such as Web pages, images and other resources, and the courseware can be distributed to another platform by export and import facilities. The exported meta-information is architecture-independent and provides a model of courseware distribution. This paper presents how to promote the distribution and sharing of Web-based e-learning courseware, where courseware can be easily retrieved by weighted keywords.

The remainder of this paper is organized as follows: Section II details e-learning systems that work with SWFs. Section III shows the outline of distributing SWF-based 
courseware to another platform. Section IV illustrates how to retrieve courseware by weighted keywords and share courseware by export and import facilities. Section V discusses the evaluation of the system. Finally, Section VI summarizes the paper.

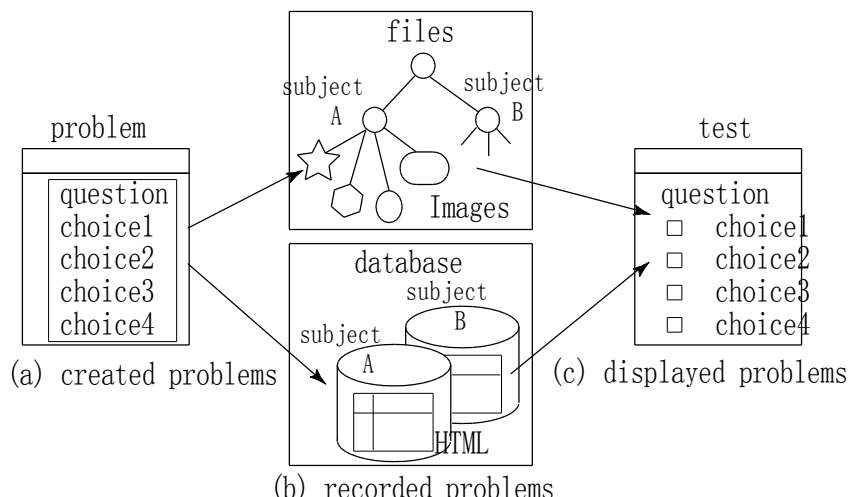

Fig. 1. Structure of SWF-based e-learning courseware.

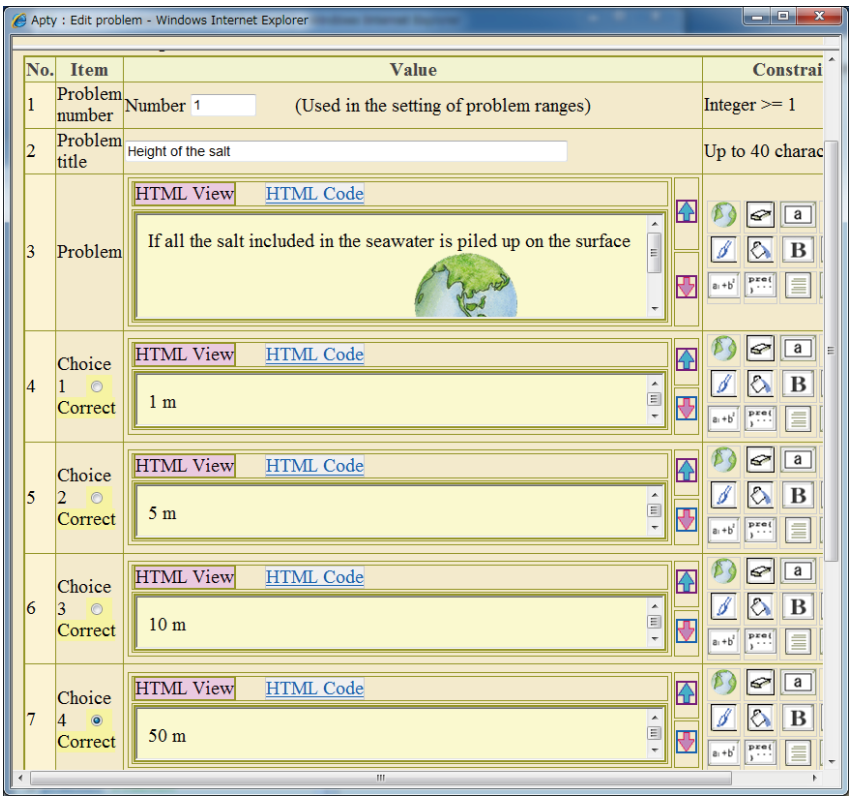

Fig. 2. A 4-choice question being created.

\section{SWF-BASED E-LEARNING SYSTEMS}

This section describes e-learning systems that work with SWFs (Sharable Web Fragments). Fig. 1 shows the system configuration of one of these types of e-learning systems. The system consists of an authoring subsystem and a learning subsystem. With the authoring subsystem, teachers can easily create some problems. For example, a problem consists of a question statement, several choices, and advice to solve this problem. These materials are created by using a Web browser and turn into HTML code. The materials may include other resources such as images and hyperlinks to some PDF files or other Web pages. The authoring subsystem stores these materials into database (hereafter DB) tables, and copies the resources to appropriate directories. That is, the courseware is stored in both of DB tables and files on the server side.

The learning subsystem will present the created problem when a test is conducted. From the contents of the DB tables, a test Web page will be automatically generated. Then, as this Web page refers to some resources in the directories on the server side by using <img src/> or < a href/> tags, those resources will be downloaded from the server side to the client side to be viewed.

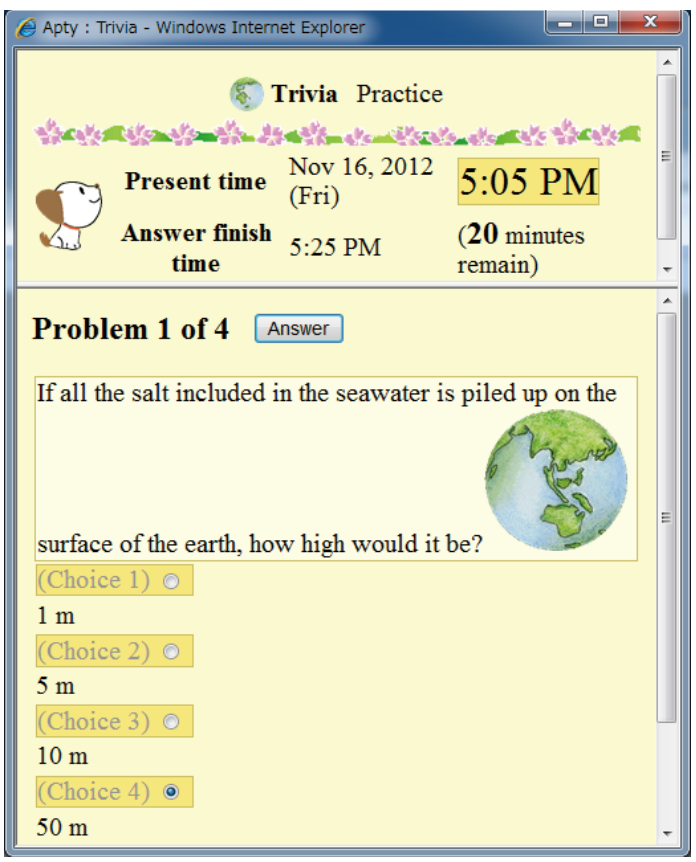

Fig. 3. A test based on the created question.

Fig. 2 demonstrates a four-choice question being created by using a Web browser, and Fig. 3 demonstrates a test which is presented with the created question. The earth image has been uploaded from the client side to the server side when it is inserted into the question statement. To conduct tests, the teachers have only to create four-choice questions. The other parts of the test Web page have been automatically generated by the learning subsystem. The teachers do not need to write any HTML code or JavaScript code.

\section{EXPORT / IMPORT AND REGISTRATION / RETRIEVAL OF SUBJECTS}

This section presents some methods for distributing SWF-based courseware to another platform.

\section{A. An SWF-Based e-Learning System}

The Apty system [12], [13] introduced here is one of the SWF-based e-learning systems. The system consists of a Web server and a DB server. The Web server accepts users' requests submitted by Web browsers. The DB server is used to store information about courseware and students' records.

In the conventional systems, users have to install these servers and set them up. It is not easy for end-users, that is, teachers to install these servers appropriately. On the other hand, this system enables teachers to install them at a time by only entering a password for an administrator. The selection of appropriate server ports and setting configuration files are all automated. To make this possible, an installation tool and an administration tool have been developed.

In the conventional systems, it takes some page transfers to reach the subjects of interest including login processes. It is also not easy for students to have an immediate understanding of what they can learn from each subject. In this system, students can view a list of all subjects without login, and they can begin practice drills of the subjects and 
enjoy games which belong to the subjects. The practice drills enable the students to acquire a better understanding of the subjects even if they are not registered for those subjects.

In the conventional systems, it has been reported that it is difficult to maintain the learning motivation because of the absence of instructors and communication with others. Students are likely to get tired of studying online courseware. To promote students' motivation, this system encourages students to compete with each other. For example, top 10 scores are shown in practice drills and exams. If students have studied subjects harder, they can gain a higher ascendancy in the games that belong to the subjects, during which they can also chat with each other.

Teachers sometimes have to develop courseware, problems (i.e. questions) and assignments to be presented in Web pages. They have to make sure that the problems they have made are all correct. They need to grade students' papers submitted for the assignments. When a new semester comes, they have to set up everything for new students. It is important to lighten the teachers' load of conducting e-learning. In this system, to make it possible to share courseware with other teachers, export and import facilities have been introduced for courseware distribution. Teachers can take a self-test of the problems they have made. In the self-test, they answer these problems in the same way as students do. This mechanism is useful to keep teachers from making wrong problems. They can also delegate the right to grade papers to someone such as assistants or graduate students. They can easily set up everything for a new semester according to a guide Web page. They do not need to be aware of DB tables for students' records.

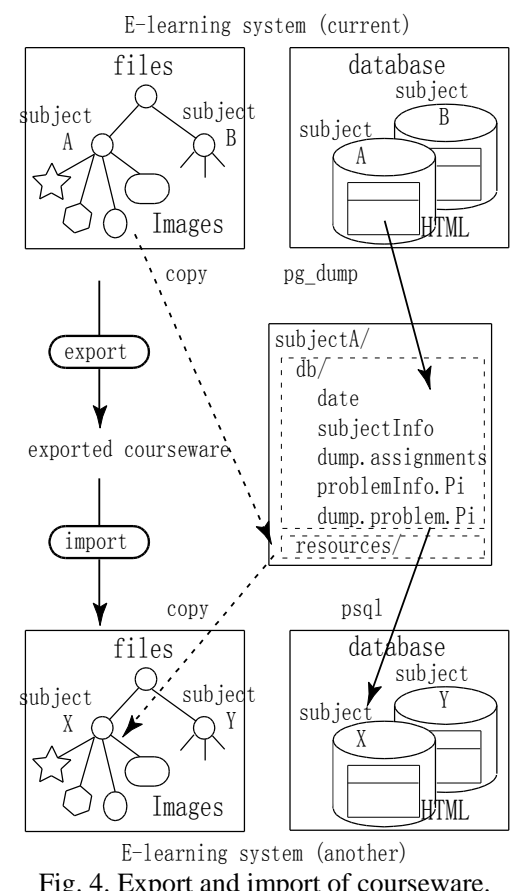

\section{B. Courseware Distribution}

The SWF-based courseware that is once exported can be imported by another SWF-based e-learning system. Fig. 4 illustrates a model of exporting and importing courseware. Among several subjects one e-learning system manages, any one of them can be exported for distribution. In the figure, for example, subject A is exported. The exported subject includes courseware materials generated from DB tables and other resources copied from directories on the server side. This exported subject will be transferred to another platform to be imported. Once the exported subject is imported by another e-learning system, this subject can be used in the same way as the other subjects that originally exist in that e-learning system.

A subject is exported into the export directory in the current e-learning system. For another e-learning system to import this subject, this exported subject needs to be located in the import directory of the e-learning system. The figure explains how subject $\mathrm{A}$ is exported and imported as subject $\mathrm{X}$ into another e-learning system.

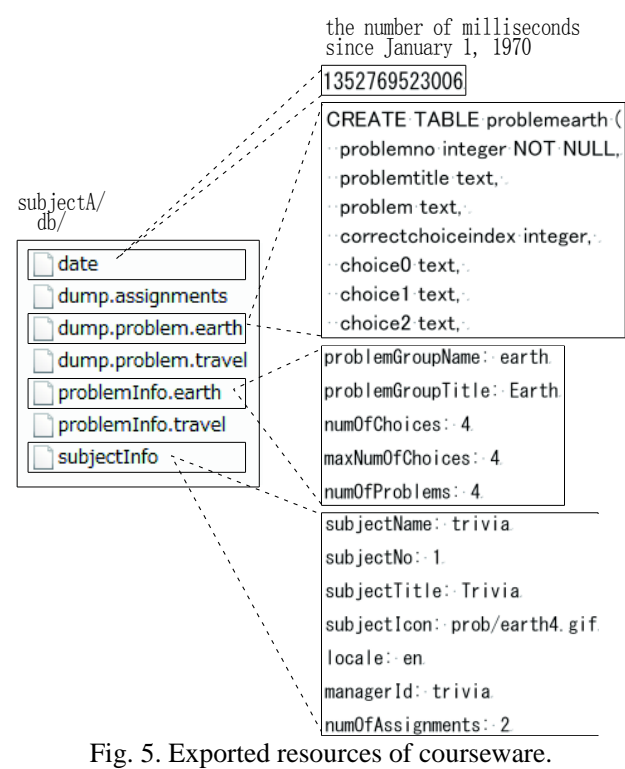

\section{Exported Materials}

An exported subject contains materials generated from DB tables by using pg_dump commands (one of PostgreSQL [14] DB commands), and resources copied from directories. Fig. 5 shows exported materials in detail. The contents of file "date" indicates the date and time when the subject was exported. They indicate the number of milliseconds since January 1, 1970. To display the date and time according to the locale selected by an administrator, the "date" file contains this type of number. The date and time will be shown as "Nov 25, 2014 (Tue) 10:18 AM" in locale "en (English)", "25-nov-2014 (mar) 10.18" in locale "it (Italian)", and "2014/11/25 (火) 10:18" in locale "ja (Japanese)".

File "dump.problem.earth" contains the psql command sequence generated by using the pg_dump command. It will be used to recover the contents of DB table "problemearth", which contains several four-choice problems. File "problemInfo.earth" contains the meta-information of DB table "problemearth", which contains problem group name "earth", problem group title "Earth", the number of choices, and so on. File "subjectInfo" contains the meta-information of subject "trivia", which contains subject name "trivia", subject title "Trivia", locale "en", and so on. These pieces of meta-information will be used as default values when the subject is imported, and then they can be changed as the administrator of this subject likes. 
The whole subject is exported at a time. On the other hand, when the exported subject is used to be imported, the whole subject, only assignments, or only problems can be imported. The exported assignments and problems will be added to the existing ones in another subject. To distribute courseware to different platforms, the contents of DB tables must be independent from those platforms. For this reason, for example, file path information is stored as URL format in the DB tables. A file path "imagelproblearth.png" in Microsoft Windows will be stored as "image/prob/earth.png".

\section{Transformation of URLs in HTML Code}

The materials of the courseware may include other resources such as images and hyperlinks to some PDF files or other Web pages. These resources are referred to by using <img src/> or < a href/> tags. The SCORM-based e-Learning systems use resources under the same directory of SCOs, which are referred to as relative path names like <img src="earth.png"/> or <a href="memo.pdf"/>. On the other hand, the SWF-based e-Learning systems can refer to any resources in the corresponding subject directories, and those resources are referred to as <img src="/apty/subjects/subjectA/image/earth.png"/> or <a href="/apty/subjects/subjectA/doc/memo.pdf"/>. When the materials are imported to another subject "subjectX", these references will be automatically transformed into <img src="/apty/subjects/subjectX/image/earth.png"/> or <a href="/apty/subjects/subjectX/doc/memo.pdf"/>.

This transformation can be automatically performed by applying a regular expression. The corresponding resource files will be copied to the appropriate locations of the subject "subjectX" directory. When problems are imported and added to a target e-learning system, if they refer to the files whose names are the same as existing files in the target e-learning system, those file names will be automatically transformed into other ones. For example, if subject "subjectX" has an image file "image/earth.png", the imported file's name will be automatically changed to "image/earth.imported.0.png". The references to this imported file will be also automatically transformed to "/apty/subjects/subjectX/image/earth.imported.0.png".

\section{E. Registration and Retrieval of Courseware by Weighted Keywords}

A courseware Web site [15] has been developed, where courseware can be registered for sharing and retrieved by anyone. Fig. 6 shows a flow of courseware registration and retrieval. To register courseware, first, its title, keywords, exported courseware archive, author's name and affiliation, and instructions about how to use the courseware are entered at this Web site. Keywords are separated with a space. The more keywords are registered, the more frequently the courseware might be retrieved later. Next, this Web site will automatically add some information like the registration date, the registration domain name, and the courseware archive's volume to the courseware information, and then store it in the courseware DB.

Registered courseware is retrieved by specifying retrieval keywords that match any part of courseware's title, keywords and author. Retrieval keywords are separated with a space. The same keyword can be specified for retrieval. If one keyword is specified two times, its weight will be double. We call this a weighted keyword. The retrieved courseware will be listed in the descending order of the number of matched retrieval keywords. Therefore, the courseware that matches weighted retrieval keywords will come first. The results of courseware retrieval can be controlled by weighted keywords. If two pieces of courseware have the same weight of keywords, the newer will come first.
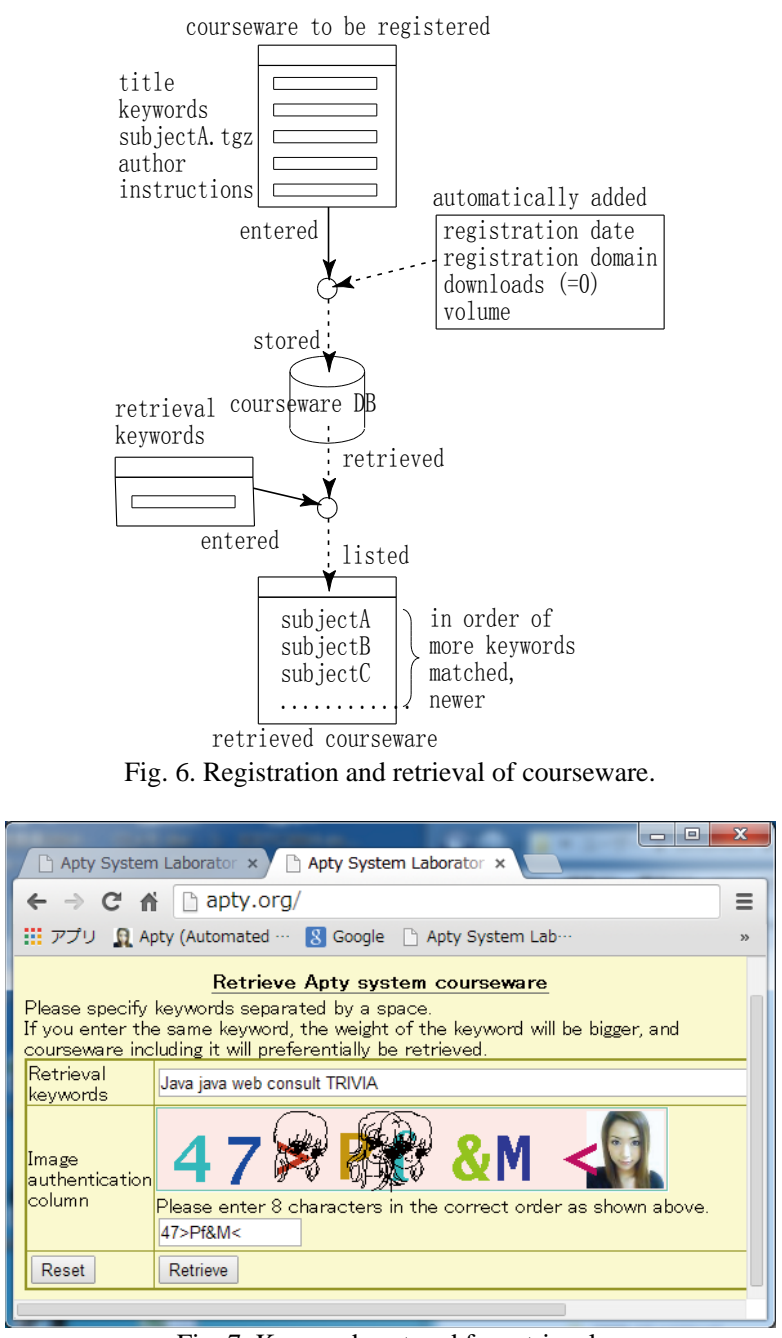

Fig. 7. Keywords entered for retrieval.

\section{EXAMPLES OF COURSEWARE DISTRIBUTION AND SHARING}

This section demonstrates user interfaces for retrieving courseware by weighted keywords and sharing courseware by export and import facilities.

\section{A. Courseware Distribution}

Fig. 7 demonstrates an example of entering keywords and Fig. 8 demonstrates retrieved results. Keywords are case-insensitive. The same keyword can be specified (like, "Java" and "java" in the figure), which has more weight, and the courseware that contains that keyword will be preferentially retrieved. The retrieval results show courseware's title, keywords, "Subject export file" button, author, registered date (with registration domain name) and the number of downloads. The parts of courseware's title, keywords and author that match the retrieval keywords will be highlighted in reverse video as shown in the figure. The 
courseware archive can be downloaded by clicking on its "Subject export file" button, and then the number of downloads will be incremented.

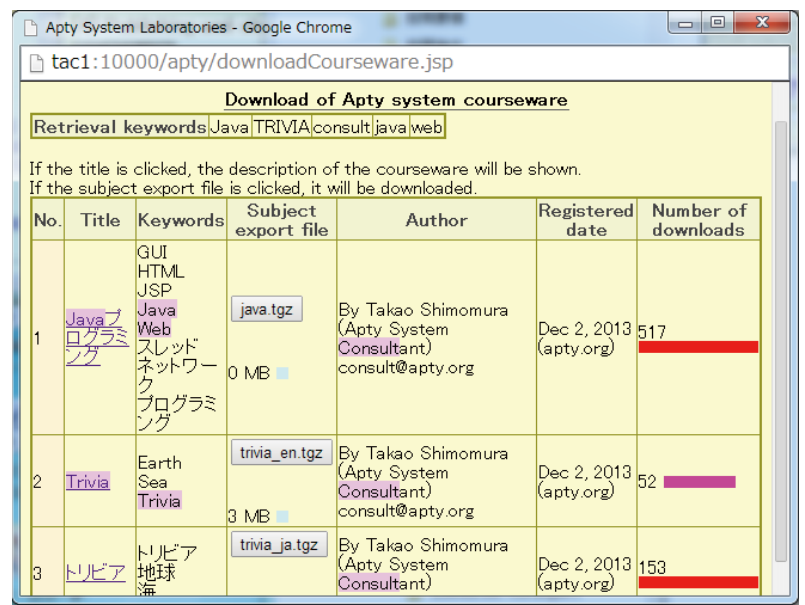

Fig. 8. Courseware retrieved with weighted keywords.

\section{B. Courseware Sharing}

Fig. 9-Fig. 11 demonstrate an example of transferring courseware from one platform to another. Fig. 9 shows the result of exporting subject "trivia". The displayed result shows that other subjects "english" and "math" have already been exported and exist in the export directory. These exported subjects will be registered in the courseware Web site described in Section IV.1. They will be then retrieved and imported into another platform.

Any of the whole subject, assignments, and problems can be imported. Fig. 10 here shows the procedure of importing problems. First, a subject is chosen, and then a set of problems in the subject will be selected. When the "Add to an existing problem group" button is clicked, as shown in Fig. 11 , the problem group can be selected to which the imported problems will be added. As some new problems are added into the existing problem group, the problem numbers of these added problems will be incremented by 10 as indicated in the figure. As a result, these new problems will become available in the target e-learning system.

\section{EVALUATION}

In SWF-based e-learning systems, everyone can easily make courseware by using a Web browser. Unlike SCORM, they are not required to have knowledge about HTML or JavaScript. In addition, the created courseware can be distributed to another e-learning system by the export and import facilities as described. Instead of these facilities, if courseware is manually transferred to another e-learning system, it will take a lot of procedures. First, DB tables need to be dumped by using pg_dump commands, and the dumped files and subject-related resource files need to be transferred to the target e-learning system by ftp or scp. In the target system, DBs will be created and DB tables will be generated from the transferred dumped files by using psql commands. Furthermore, URLs of HTML code contained in the DB tables need to be transformed. The export and import facilities have saved these laborious procedures. In addition, the courseware Web site can be used to promote courseware distribution and sharing.

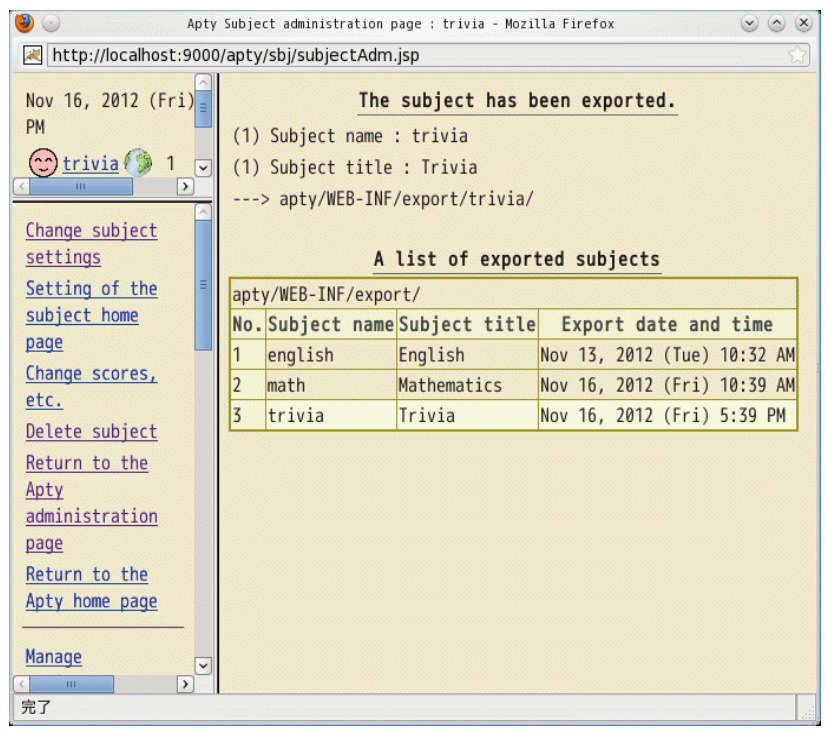

Fig. 9. Example of subject export.

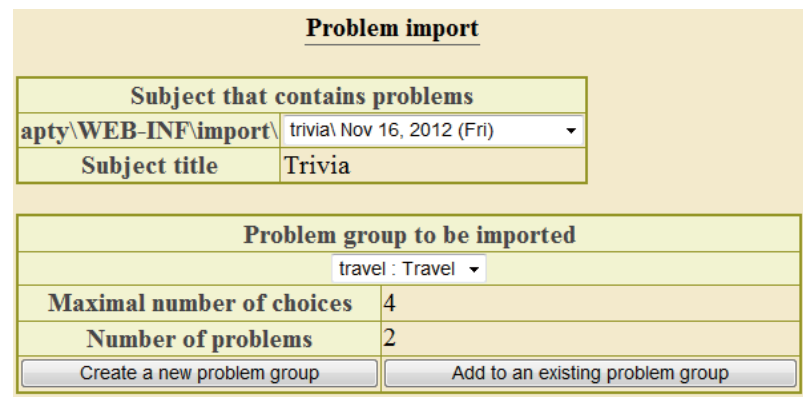

Fig. 10. Example of problem import.

\begin{tabular}{|c|c|c|}
\hline \multicolumn{3}{|c|}{ Additional import of problems } \\
\hline \multirow{2}{*}{ Subject } & Subject name & trivia \\
\hline & Subject title & Trivia \\
\hline \multirow{4}{*}{ Problem group } & Problem group name & travel \\
\hline & Problem group title & Travel \\
\hline & Maximal number of choic & $\mathrm{s} 4$ \\
\hline & Number of problems & 2 \\
\hline
\end{tabular}

Problem group to which the imported problems will be added

\begin{tabular}{|l|l|l|l|}
\hline No. & \multicolumn{1}{|c|}{ Item } & \multicolumn{1}{c|}{ Value } & \multicolumn{1}{c|}{ Constraints } \\
\hline 1 & Problem group name & travel : Travel & \\
\hline 2 & Number of choices & 4 & \\
\hline 3 & Maximal problem number & 2 & \\
\hline 4 & Increment added to problem numbers & 10 & Integer $>=2$ \\
\hline \multicolumn{2}{|l|}{ Display current settings } & \multicolumn{2}{|c|}{ Import problems } \\
\hline
\end{tabular}

Fig. 11. Example of additional import of selected problems.

\section{CONCLUSION}

This paper has presented export and import methods for courseware sharing. In SWF-based e-learning systems, courseware can be easily created by using a Web browser without any knowledge about HTML or JavaScript. The courseware can be easily distributed to another e-learning system. Because the exported subject is architecture-independent, it will be useful even when courseware is distributed to an e-learning system that has a different architecture. The e-learning system this paper has presented will be available at http://apty.org/. Distribution and sharing of courseware will be also available at the Web site above. 


\section{REFERENCES} [1] M. Dougiamas.
http://moodle.org/.

(2012). Moodle. [Online]. Available:

[2] E. O' Neill, N. Stevens, E. Clarke, P. Cox, B. O' Malley, and H. Humphreys, "Use of e-learning to enhance medical students' understanding and knowledge of healthcare-associated infection prevention and control," Journal of Hospital Infection, 2011.

[3] C. Huang, S. Chin, L. Hsin, J. Hung, and Y. Yu, "A web-based e-learning platform for physical education," Journal of Networks, vol. 6, no. 5, pp. 721-727, 2011.

[4] W. Song, S. Cao, B. Yang, K. Song, and C. Wu, "Development and application of an autonomous learning system for engineering graphics education," I. J. Modern Education and Computer Science, vol. 1, pp. 31-37, 2011.

[5] N. Joshi, "Semantic web-driven e-learning system," DESIDOC Journal of Library \& Information Technology, vol. 31, no. 4, pp. 213-216, 2011.

[6] G. Wu and Y. Wang, "Research on e-learning system prototype based on semantic web service technology," Computing and Intelligent Systems, pp. 247-254, 2011.

[7] AICC. (2012). Aviation Industry Computer-Based Training Committee [Online]. Available: http://www.aicc.org/

[8] C. Qu and W. Nejdl, "Towards interoperability and reusability of learning resources: A SCORM-conformant courseware for computer science education," in Proc. the 2nd IEEE International Conference on Advanced Learning Technologies (IEEE ICALT 2002), 2002.

[9] SCORM. (2012). Sharable Content Object Reference Model. [Online]. Available: http://www.adlnet.org/.

[10] Q. Li and J. Zhang, "Preliminary Study on Extracting SCORM Contents to MOODLE," in Proc. 2011 Third Pacific-Asia Conference on Circuits, Communications and System (PACCS), 2011, pp. 1-3.

[11] R. Harper, "Delivery of courseware, tutorials and formative assessment with WebOL," International Journal of Innovation in Science and Mathematics Education (formerly CAL-laborate International), vol. 11, no. 1,2012

[12] T. Shimomura, A. Montanaro et al., Easy, Enjoyable, Effective, e-Learning, Nova Science Publishers, Inc., 2008.

[13] A. Montanaro, K. Ikeda, and T. Shimomura, "Game-Oriented Stimulating E-Learning," in Proc. 6th International Technology, Education and Development Conference (INTED2012), 2012, pp. 4221-4228.

[14] The PostgreSQL Global Development Group. (2014). PostgreSQL. [Online]. Available: http://www.postgresql.org/.

[15] Apty System Consultants. (2014). Distribution and Sharing of Apty-based Courseware. [Online]. Available: http://apty.org/.
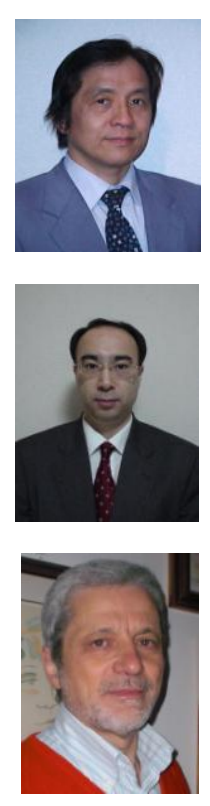

Takao Shimomura is a professor of the Dept. of Information Science and Intelligent Systems at the University of Tokushima in Japan. He obtained his PhD in computer science from Tokyo Institute of Technology in Japan. His research interests include software design automation, program visualization, and automated debugging.

Kenji Ikeda is an associate professor of the Dept. of Information Science and Intelligent Systems at the University of Tokushima in Japan. He received a BE, $\mathrm{ME}$ and $\mathrm{PhD}$ from the University of Tokyo. His research interests include adaptive control and system identification.

propagation of discon mechanical systems. 